\title{
Chloroplast transit peptides often require downstream unstructured sequence in Chlamydomonas reinhardtii
}

1 Caspari, OD $^{1,2}$

$2{ }^{1}$ Affiliation: Chloroplast Biology and Light Sensing in Microalgae research group (UMR7141),

3 Institut de Biologie Physico-Chimique (CNRS/Sorbonne Université), 13 Rue Pierre et Marie Curie,

475005 Paris, France

$5 \quad{ }^{2}$ Current address: Stress Adaptation and Metabolism in Enterobacteria research group, Department of

6 Microbiology, Pasteur Institute, 28 Rue du Docteur Roux, 75015 Paris, France

* Correspondence:

8 Caspari, OD

9 odc20@cantab.net

10 Keywords: disordered protein, protein import, chloroplast, targeting peptide, transit peptide, 11 presequence, Chlamydomonas reinhardtii

\section{Abstract}

13 The N-terminal sequence stretch that defines subcellular targeting for most nuclear encoded chloroplast proteins is usually considered identical to the sequence that is cleaved upon import. Yet here this study shows that for nine out of ten tested Chlamydomonas chloroplast transit peptides, additional sequence past the cleavage site is required to enable chloroplast targeting. Using replacements of native post-cleavage residues with alternative sequences points to a role for unstructured sequence at mature protein $\mathrm{N}$-termini.

\section{Introduction}

Chloroplasts derive from once free-living cyanobacteria and retain genomes of their own, yet the vast majority of genes coding for plastid proteins are located in the nucleus (Shi and Theg, 2013). Proteins are expressed in the cytosol as preproteins containing N-terminal chloroplast targeting peptides (cTP), which direct import into the chloroplast (Chotewutmontri et al., 2017). Upon import, the Nterminus of the preprotein is cut off at a defined cleavage site and degraded (Teixeira and Glaser, 2013; Kmiec et al., 2014).

The cTP, i.e. the sequence both necessary and sufficient to generate chloroplast targeting, is generally assumed to coincide with the sequence stretch that is cleaved off. It is this cleaved presequence that has been studied for decades, with a well-documented tripartite structure and multiple sequence elements now recognized to contribute to targeting (von Heijne et al., 1989; Chotewutmontri et al., 2017; Caspari and Lafontaine, 2021). Yet the cleavage site may not always be a reliable guide for defining the cTP. Parts of the mature protein N-terminus have been recognized before as a requirement for efficient targeting in higher plants (Bionda et al., 2010; Rolland et al., 2016; Shen et al., 2017). Bionda et al. (2010) described a length requirement of 55-60 amino acids for Arabidopsis thaliana, and proposed that in the $\sim 50 \%$ of chloroplast targeted proteins where the cleavable part of the cTP is shorter, residues $\mathrm{C}$-terminal of the cleavage site contribute unstructured sequence towards targeting. There are even examples of import through the canonical TOC/TIC system of proteins 
37 lacking a cleavable N-terminal presequence altogether (Chang et al., 2014), implying the entire

38 targeting information is present in the mature protein.

39 It was noted decades ago that cleavable cTPs are generally shorter in the model green alga

40 Chlamydomonas reinhardtii compared to higher plants based on the placement of cleavage sites

41 (Franzén et al., 1990), suggesting there may be a role for mature protein N-termini. In

42 Chlamydomonas, the Rubisco activase (RBCA) cTP has recently been found to require post-cleavage

43 site residues to target a fluorescent reporter protein to the chloroplast (Garrido et al., 2020), in line

44 with earlier work regarding Rubisco small subunit (RBCS) cTP from Chlamydomonas (Razzak et al.,

45 2017) and higher plants (Comai et al., 1988). This present study revisits the role of post-cleavage site

46 residues, and finds that substantial stretches of unstructured sequence appear to be required to enable

47 targeting by most Chlamydomonas cTPs.

\section{$48 \quad 3 \quad$ Materials and Methods}

49 Constructs were Gibson assembled as described in (Garrido et al., 2020) based on pMO611, a variant

50 of published pMO449 (Onishi and Pringle, 2016) where an upstream start codon was mutated to

51 CTG. Sequences inserted upstream of Venus were amplified from Chlamydomonas genomic DNA extracted from wild-type strain T222+, with the following exceptions: construct 'RBCS-cTP +23 ' was amplified from cDNA generated from T222+ to avoid introducing an intron not present in the other RBCS-cTP constructs; the G-rich artificial linker sequence was amplified from plasmid CrRaf1pJHL encoding Raf1-strep (Wietrzynski et al., 2021). Corresponding amino acid sequences are given in Table 1. Constructs were verified by sequencing (Eurofins). Transformants were obtained and microscopy performed as described before (Garrido et al., 2020).

\section{$4 \quad$ Results}

Native cTPs were tested by inserting the respective genomic sequences upstream of a Venus fluorescent reporter in a bicistronic expression vector (Onishi and Pringle, 2016; Caspari, 2020). Neither RBCA nor RBCS cTPs enabled import into the chloroplast (Fig. 1A,B), instead showing Venus fluorescence from across the cytosol or from within globular structures outside the chloroplast respectively. Given that successful targeting using the RBCS-cTP including 23 residues downstream of the cleavage site had previously been reported in Chlamydomonas (Razzak et al., 2017) and higher plants (Comai et al., 1988) and extending the RBCA-cTP by 23 residues had equally restored targeting (Garrido et al., 2020), a series of successive extensions by 3 (Fig. 1C,D), 5 (Fig. 1E,F), 10 (Fig. $1 \mathrm{G}, \mathrm{H}$ ) and 23 residues (Fig. 1I,J) was prepared. The $+3,+5$ and +10 constructs of both RBCAcTP and RBCS-cTP all show significant Venus fluorescence from outside the chloroplast (Fig. 1C$\mathrm{H}$ ), and only the RBCA-cTP+10 construct also shows some partial localization of Venus to the chloroplast (Fig. 1G). By contrast, the +23 constructs show clear accumulation of Venus inside the chloroplast (Fig. 1I,J), strikingly visible as fluorescence from inside the pyrenoid, a proteinaceous structure within the chloroplast that generates a visible dip in chlorophyll fluorescence (Mackinder et al., 2017). Note that the RBCA-cTP 23 construct contains a pyrenoid-localisation motif (Meyer et al., 2020). By contrast, mitochondrial targeting peptides (mTPs) from carbonic anhydrase 4 (CAH4) and $\gamma$-carbonic anhydrase 2 (CAG2) show mitochondrial targeting, evident as co-localisation of Venus and MitoTracker fluorescence, in the absence of any post-cleavage site residues (Fig. 1K,L). CAG2-mTP shows some remaining signal from the cytoplasm, which persists when the construct is extended by 23 residues past the cleavage site (Garrido et al., 2020). 
79

80

81

82

83

84

85

86

87

88

89

90

91

92

93

94

95

96

97

98

99

100

101

102

103

104

105

106

107

108

109

110

111

112

113

114

115

116

117

118

119

120

121

122

123

To test whether the presence of a relatively long stretch of mature protein is a general requirement rather than an exception concerning specifically RBCA and RBCS, a further eight cTPs were assayed for import (Fig. 2). These cTPs were each extended by 5 residues beyond the cleavage site to ensure any lack of import was not an artifact arising from a missing cleavage site, for which several residues past the cleavage site seem to be important (Tardif et al., 2012). Only one of the tested cTP +5 constructs, that of PSAD, generates chloroplast localized Venus signal (Fig. 2A-H). Chloroplast targeting with the PSAD-cTP also works in the absence of any post-cleavage site residues (Fig. 2I).

To follow up the hypothesis that post-cleavage site residues contribute unstructured sequence towards chloroplast targeting, four constructs were prepared that each extend the RBCA-cTP +5 by different sequences (Fig. 3). Five mature RBCA residues were kept to ensure the cleavage site would be maintained intact. Three constructs contained unstructured sequence downstream (A-C) and were expected to generate chloroplast targeting, whereas a fourth (D) contained a $\alpha$-helical stretch that was expected to impede chloroplast import. Two of the unstructured sequences as well as the helical stretch were copied from Chlamydomonas nuclear genes encoding chloroplast-localized proteins EPYC1 (also known as LCI5), VIPP1 and RBCXa respectively. In each of these cases, sequence was chosen from the $\mathrm{C}$-terminal half of the protein, to avoid any sequence that might contribute to the cTPs of these proteins. In addition, a standard G-rich linker sequence, such as is commonly used to join elements when generating fusion proteins in molecular biology, was used to test whether an artificial flexible sequence could substitute for a native unstructured one.

Of these, natively unstructured sequences from the third repeat of intrinsically disordered EPYC1 and the disordered C-terminus of VIPP1 generated chloroplast localization of the Venus reporter (Fig. $3 \mathrm{~A}, \mathrm{~B})$. Note that the VIPP1-derived sequence also shows additional Venus signal emanating from cytosolic structures (Fig. 3B). By contrast, both the artificial flexible linker and the C-terminal $\alpha$ helix of RBCXa resulted in only a small amount of Venus localizing to the chloroplast, with a majority of signal emanating from structures outside the chloroplast (Fig. 3C,D).

\section{Discussion}

By definition, targeting peptides comprise the sequence that is both necessary for targeting a native cargo protein to its subcellular destination, and sufficient to redirect a heterologously expressed cargo to the same location (Chotewutmontri et al., 2017). The present study demonstrates that in Chlamydomonas, the amino-terminal sequence required to specify chloroplast targeting exceeds the cleavable part for nine out of ten tested cTPs. Care was taken to make the set of studied cTPs as representative as possible within the limited choice of cTPs with a well-characterized cleavage site (Terashima et al., 2011; Tardif et al., 2012). In particular, studied cTPs were chosen to include proteins related to photosynthesis (e.g. RBCA, RBCS, PSAD) as well as to plastid housekeeping (e.g. ribosomal proteins PRPL4, PSRP3), as import of these groups may be mediated by different receptors at the translocon (Chotewutmontri et al., 2017). The cleavage site should thus no longer be considered a reliable guide for identifying the $\mathrm{N}$-terminal stretch containing all chloroplast targeting information.

The reduced length of algal cTPs (Franzén et al., 1990) may partly be accounted for by specific sequence elements required for targeting in plant cTPs being reduced in Chlamydomonas, such as a TOC-interacting FGLK motif (Chotewutmontri et al., 2017; Razzak et al., 2017; Caspari et al., 2021). Yet the requirement for post-cleavage site residues implies that the amino-terminal sequences contributing to targeting in Chlamydomonas are in fact longer than recognized to date, with sequence elements that play a role in import present past the cleavage site just as in short plant cTPs (Caspari et al., 2021). With lengths of 56 and 60 residues respectively, the import competent $\mathrm{RBCA}-\mathrm{cTP}+23$ 
124 and RBCS-cTP +23 constructs mirror the $\sim 55-60$ amino acid requirement Arabidopsis cTPs (Bionda

125 et al., 2010). In this sense, algal cTPs may not be shorter than their plant counterparts after all.

126 Previous studies suggested that the contribution of mature protein N-termini towards targeting may

127 simply be a supply of unstructured sequence (Bionda et al., 2010; Shen et al., 2017). Bionda et al.

128 (2010) found that using tightly folded titin as cargo protein prevented chloroplast import by short

129

130

131 cTPs unless first denatured. Shen et al., (2017) found that 20 residues of unfolded sequence downstream of the cTP cleavage site were required to target two E. coli proteins into rice

132

133

134

135

136 chloroplasts. While less tightly folded than titin, the Venus fluorescent reporter used as cargo protein in the present study contains a short, tightly folded $\alpha$-helix at the N-terminus (Rekas et al., 2002), which may conceivably impair import in the absence of an unfolded spacer sequence. In principle, the chloroplast import machinery is capable of unfolding any protein for post-translational import, driven by an ATP-powered motor complex in the chloroplast stroma (Chotewutmontri et al., 2017). However, initiation of import requires the cTP to reach across both TOC and TIC passively before being able to contact stromal motor proteins. It is this initiation step that is most likely disrupted by the presence of structured elements too close to the N-terminus (Bionda et al., 2010). Mitochondrial import lacks similar constraints, as mitochondrial targeting peptides are guided across the outer membrane translocase using interactions with increasing affinity (Schatz, 1997; Fukasawa et al., 2017), and then make use of the proton motive force across the inner membrane to power import even before contacting the matrix-localized PAM complex that uses ATP to energize further import (Martin et al., 1991; Wiedemann and Pfanner, 2017). On the other hand, Razzak et al. (2017) report the presence of a specific motif $(\mathrm{Q} \mid \mathrm{MMVW})$ required for targeting in the Chlamydomonas RBCScTP that spans the cleavage site (Schmidt et al., 1979), suggesting post-cleavage site sequence elements may also play a more active role in specifying targeting.

147 Here, we found that chloroplast import by RBCA-cTP could indeed be restored using unstructured 148 sequences taken from chloroplast proteins EPYC1 and VIPP1 as post-cleavage site spacers. EPYC1 is an intrinsically disordered protein comprised of four near-identical internal repeats that act as linker protein within the chloroplast pyrenoid (Mackinder et al., 2016), a proteinaceous structure within the chloroplast that is central to the algal carbon concentrating mechanism (Caspari et al., 2017). VIPP1 plays a role in the biogenesis of thylakoid membranes and has an unstructured Cterminus (Zhang et al., 2016; Theis et al., 2019). C-terminal sequences from these proteins are not expected to contain any specific targeting motifs, thus restoration of targeting lends further support to the importance of unstructured sequence at the N-terminus. In the same vain, copying an inherently structured sequence from the most C-terminal helix of RBCXa (Bracher et al., 2015) largely

157 prevented chloroplast targeting (Fig. 3D).

An overall picture thus emerges of short algal cTPs up to the cleavage site containing the information for chloroplast targeting, but relying on additional unstructured sequence at mature protein $\mathrm{N}$-termini to allow import to be successfully initiated. The importance of sufficient length and the presence of unstructured sequence elements for chloroplast targeting was equally evident in recent work aimed at retracing likely steps in the evolution of targeting peptides from putative antimicrobial origins (Caspari et al., 2021). Additional complexities likely exist, as several observations point to further factors being at play: A G-rich linker failed to restore chloroplast import beyond what had been seen for the RBCXa helix (Fig. 3C), suggesting that this artificial flexible sequence differs in important ways from native unstructured sequences. Furthermore, the very long PSRP3-cTP +5 construct (89 residues) failed to target whereas the relatively short PSAD-cTP (35 residues) achieved targeting, suggesting additional factors are in play beyond cTP length. The case of PSAD is particularly intriguing, as this cTP should be too short to reach into the stroma passively, yet appears to 
170

171

172

173

174

175

176

177

178

179

180

181

182

183

184

185

186

187

188

189

190

191

192

193

194

195

196

197

198

199

200

201

202

203

204

205

circumvent the impedance of a folded Venus cargo. Presumably, the cargo protein is kept in an unfolded state prior to import, e.g. through recruitment of chaperones interacting with the cTP, or perhaps through co-translational import following polysome recruitment to the chloroplast envelope by this cTP. Either way, for the purposes of sending heterologous proteins to the chloroplast (Crozet et al., 2018), the PSAD-cTP is likely the best choice among Chlamydomonas cTPs studied so far to ensure targeting without a need for additional sequence at the mature protein N-terminus.

\section{Conflict of Interest}

The author declares that the research was conducted in the absence of any commercial or financial relationships that could be construed as a potential conflict of interest. The funders had no role in the design of the study; in the collection, analyses, or interpretation of data; in the writing of the manuscript, or in the decision to publish the results.

\section{$7 \quad$ Data availability statement}

The original contributions presented in the study are included in the article/supplementary material, further inquiries can be directed to the corresponding author/s.

\section{Author Contributions}

Conceptualisation, Methodology, Investigation, Analysis, Interpretation, Visualisation and Writing: ODC

\section{$9 \quad$ Funding}

Financial support from the Rothschild Foundation, LabEx Dynamo (ANR-LABX-011) and the ChloroMitoRAMP grant (ANR-19-CE13-0009) to ODC, as well as annual funding from CNRS and Sorbonne University to UMR7141, is gratefully acknowledged.

\section{Acknowledgments}

I would like to thank Francis-André Wollman for critical reading of the manuscript, as well as Richard Kuras and Yves Choquet for helpful discussions and advice. Katia Wostrikoff kindly provided plasmid CrRafl-pJHL from which the G-rich linker sequence was amplified. Thank you also to Stéphane Lemaire and Pierre Crozet for suggesting that I should try PSAD-cTP.

\section{References}

Bionda, T., Tillmann, B., Simm, S., Beilstein, K., Ruprecht, M., and Schleiff, E. (2010). Chloroplast import signals: The length requirement for translocation in vitro and in vivo. J. Mol. Biol. 402, 510-523. doi:10.1016/j.jmb.2010.07.052.

Bracher, A., Hauser, T., Liu, C., Hartl, F. U., and Hayer-Hartl, M. (2015). Structural analysis of the rubisco-assembly chaperone RbcX-II from Chlamydomonas reinhardtii. PLoS One 10, 1-17. doi:10.1371/journal.pone.0135448.

Caspari, O. D. (2020). Introduction of a leaky stop codon as molecular tool in Chlamydomonas reinhardtii. PLoS One 15, e0237405. doi:10.1371/journal.pone.0237405.

Caspari, O. D., Garrido, C., Choquet, Y., Wollman, F.-A., and Lafontaine, I. (2021). Retracing the 
evolution from antimicrobial to targeting peptides. bioRxiv.

Caspari, O. D., and Lafontaine, I. (2021). The role of antimicrobial peptides in the evolution of endosymbiotic protein import. PLoS Pathog. 17, e1009466. doi:10.1371/journal.ppat.1009466.

Caspari, O. D., Meyer, M. T., Tolleter, D., Wittkopp, T. M., Cunniffe, N. J., Lawson, T., et al. (2017). Pyrenoid loss in Chlamydomonas reinhardtii causes limitations in CO2 supply, but not thylakoid operating efficiency. J. Exp. Bot. 68, 3903-3913. doi:10.1093/jxb/erx197.

Chang, W. L., Soll, J., and Bölter, B. (2014). A new member of the psToc159 family contributes to distinct protein targeting pathways in pea chloroplasts. Front. Plant Sci. 5, 1-14.

214 doi:10.3389/fpls.2014.00239.

Comai, L., Larson-Kelly, N., Kiser, J., Mau, C. J., Pokalsky, A. R., Shewmaker, C. K., et al. (1988).

Chotewutmontri, P., Holbrook, K., and Bruce, B. D. (2017). Plastid Protein Targeting: Preprotein Recognition and Translocation. Int. Rev. Cell Mol. Biol. 330, 227-294. doi:10.1016/bs.ircmb.2016.09.006. Chloroplast transport of a ribulose bisphosphate carboxylase small subunit-5-enolpyruvyl 3phosphoshikimate synthase chimeric protein requires part of the mature small subunit in addition to the transit peptide. J. Biol. Chem. 263, 15104-15109. doi:10.1016/s00219258(18)68151-7.

Crozet, P., Navarro, F., Willmund, F., Mehrshahi, P., Bakowski, K., Lauersen, K., et al. (2018). Birth of a Photosynthetic Chassis: a MoClo Toolkit Enabling Synthetic Biology in the Microalga Chlamydomonas reinhardtii. ACS Synth. Biol. 7, 2074-2086. doi:10.1021/acssynbio.8b00251.

Franzén, L.-G. G., Rochaix, J.-D. D., and von Heijne, G. (1990). Chloroplast transit peptides from the green alga Chlamydomonas reinhardtii share features with both mitochondrial and higher plant chloroplast presequences. FEBS Lett. 260, 169-172. doi:10.1016/0014-5793(90)80094-Y.

Fukasawa, Y., Oda, T., Tomii, K., and Imai, K. (2017). Origin and Evolutionary Alteration of the Mitochondrial Import System in Eukaryotic Lineages. Mol. Biol. Evol. 34, 1574-1586. doi:10.1093/molbev/msx096.

Garrido, C., Caspari, O. D., Choquet, Y., Wollman, F. A., and Lafontaine, I. (2020). Evidence Supporting an Antimicrobial Origin of Targeting Peptides to Endosymbiotic Organelles. Cells 9, 1795. doi:10.3390/cells9081795.

Kmiec, B., Teixeira, P. F., and Glaser, E. (2014). Shredding the signal : targeting peptide degradation in mitochondria and chloroplasts. Trends Plant Sci. 19, 771-778. doi:10.1016/j.tplants.2014.09.004.

Mackinder, L. C. M., Chen, C., Leib, R. D., Patena, W., Blum, S. R., Rodman, M., et al. (2017). A Spatial Interactome Reveals the Protein Organization of the Algal CO2-Concentrating Mechanism. Cell 171, 133-147. doi:10.1016/j.cell.2017.08.044.

Mackinder, L. C. M., Meyer, M. T., Mettler-Altmann, T., Chen, V. K., Mitchell, M. C., Caspari, O. D., et al. (2016). A repeat protein links Rubisco to form the eukaryotic carbon concentrating 
organelle. Proc. Natl. Acad. Sci. USA 113, 5958-5963. doi:10.1073/pnas.1522866113.

245

246
Martin, J., Mahlke, K., and Pfanner, N. (1991). Role of an energized inner membrane in mitochondrial protein import: $\Delta \Psi$ drives the movement of presequences. J. Biol. Chem. 266, 18051-18057. doi:10.1016/s0021-9258(18)55235-2.

Meyer, M., Itakura, A., Patena, W., Wang, L., He, S., Emrich-Mills, T., et al. (2020). Assembly of the algal CO2-fixing organelle, the pyrenoid, is guided by a Rubisco-binding motif. Sci. Adv. 6, eabd2408. doi:10.1126/sciadv.abd2408.

Onishi, M., and Pringle, J. R. (2016). Robust Transgene Expression from Bicistronic mRNA in the Green Alga Chlamydomonas reinhardtii. G3 (Bethesda). 6, 4115-4125. doi:10.1534/g3.116.033035.

Razzak, A., Lee, D. W., Yoo, Y., and Hwang, I. (2017). Evolution of rubisco complex small subunit transit peptides from algae to plants. Sci. Rep. 7, 9279. doi:10.1038/s41598-017-09473-x.

Rekas, A., Alattia, J. R., Nagai, T., Miyawaki, A., and Ikura, M. (2002). Crystal structure of venus, a yellow fluorescent protein with improved maturation and reduced environmental sensitivity. $J$. Biol. Chem. 277, 50573-50578. doi:10.1074/jbc.M209524200.

Rolland, V., Badger, M. R., and Price, G. D. (2016). Redirecting the Cyanobacterial Bicarbonate Transporters BicA and SbtA to the Chloroplast Envelope: Soluble and Membrane Cargos Need Different Chloroplast Targeting Signals in Plants. Front. Plant Sci. 7, 185. doi:10.3389/fpls.2016.00185.

Schatz, G. (1997). Just follow the acid chain. Nature 388, 121. doi:10.1038/40510.

Schmidt, G. W., Devillers-thiery, A., Desruisseaux, H., Blobel, G., and Chua, N. (1979). NH2 Terminal Amino Acid Sequences of Precursor and Mature Forms of the Small Subunit From Chlamydomonas Reinhardtii. 83.

Shen, B. R., Zhu, C. H., Yao, Z., Cui, L. L., Zhang, J. J., Yang, C. W., et al. (2017). An optimized transit peptide for effective targeting of diverse foreign proteins into chloroplasts in rice. Sci. Rep. 7, 1-12. doi:10.1038/srep46231.

Shi, L. X., and Theg, S. M. (2013). The chloroplast protein import system: From algae to trees. Biochim. Biophys. Acta - Mol. Cell Res. 1833, 314-331. doi:10.1016/j.bbamcr.2012.10.002.

Tardif, M., Atteia, A., Specht, M., Cogne, G., Rolland, N., Brugière, S., et al. (2012). Predalgo: A new subcellular localization prediction tool dedicated to green algae. Mol. Biol. Evol. 29, 36253639. doi:10.1093/molbev/mss178.

Teixeira, P. F., and Glaser, E. (2013). Processing peptidases in mitochondria and chloroplasts. Biochim. Biophys. Acta - Mol. Cell Res. 1833, 360-370. doi:10.1016/j.bbamcr.2012.03.012.

Terashima, M., Specht, M., and Hippler, M. (2011). The chloroplast proteome: A survey from the Chlamydomonas reinhardtii perspective with a focus on distinctive features. Curr. Genet. 57, 151-168. doi:10.1007/s00294-011-0339-1. 
Theis, J., Gupta, T. K., Klingler, J., Wan, W., Albert, S., Keller, S., et al. (2019). VIPP1 rods engulf membranes containing phosphatidylinositol phosphates. Sci. Rep. 9, 1-11. doi:10.1038/s41598019-44259-3.

von Heijne, G., Steppuhn, J., and Herrmann, R. G. (1989). Domain structure of mitochondrial and chloroplastic targeting peptides. Eur. J. Biochem. 180, 535-545.

Wiedemann, N., and Pfanner, N. (2017). Mitochondrial Machineries for Protein Import and Assembly. Annu. Rev. Biochem. 86, 685-714. doi:10.1146/annurev-biochem-060815-014352.

Wietrzynski, W., Traverso, E., Wollman, F.-A., and Wostrikoff, K. (2021). The state of oligomerization of Rubisco controls the rate of synthesis of the Rubisco large subunit in Chlamydomonas reinhardtii . Plant Cell, 1-22. doi:10.1093/plcell/koab061.

Zhang, L., Kondo, H., Kamikubo, H., Kataoka, M., and Sakamoto, W. (2016). VIPP1 has a disordered C-terminal tail necessary for protecting photosynthetic membranes against stress. Plant Physiol. 171, 1983-1995. doi:10.1104/pp.16.00532. 


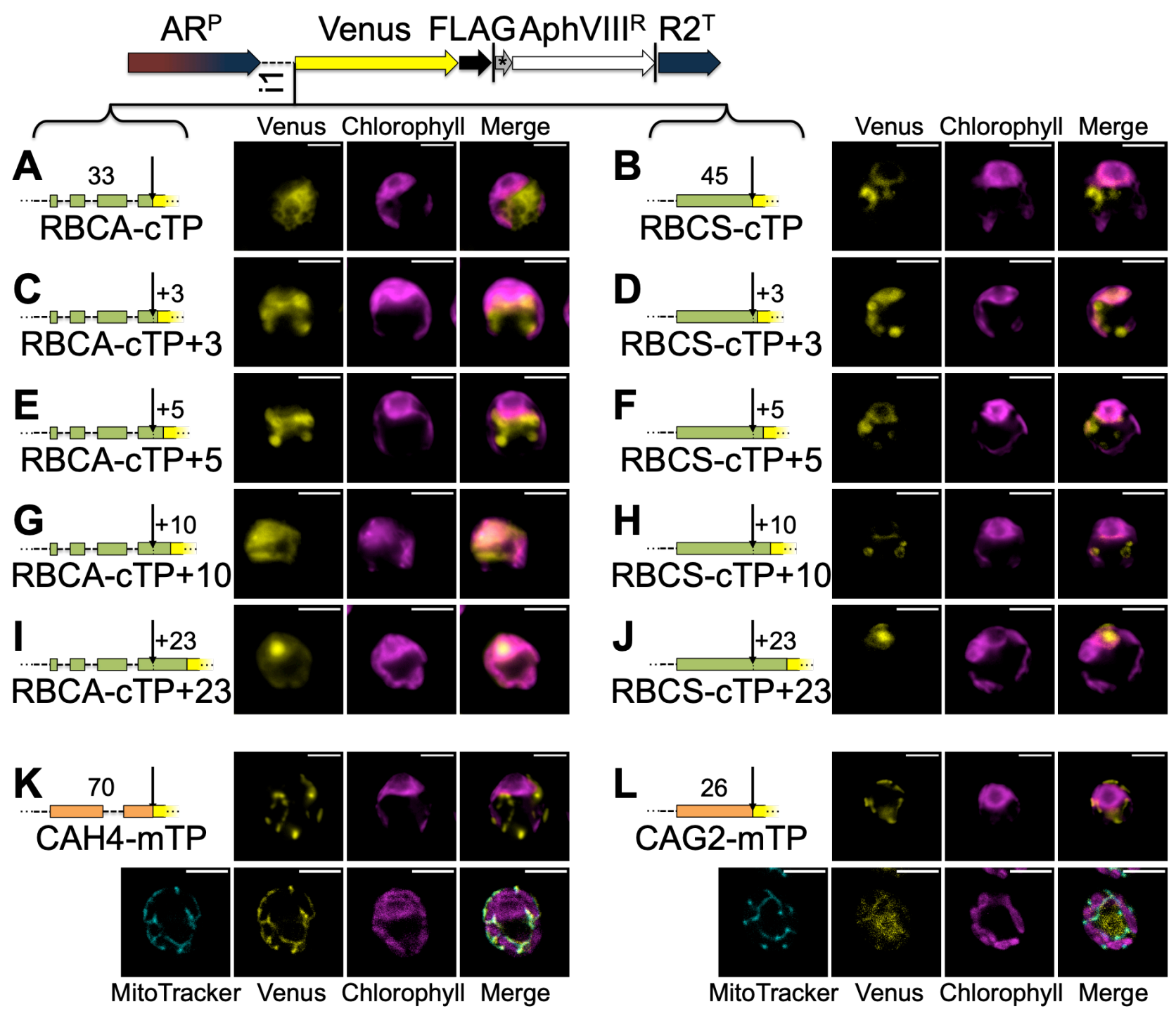

Fig. 1 - A stretch of post-cleavage site residues is necessary for chloroplast targeting peptides. Constructs were generated by inserting candidate peptides upstream of Venus in a bicistronic expression vector made of the following components: $\mathrm{AR}^{\mathrm{P}}$ : Hybrid HSP70a-RBCS2 promoter, i1: RBCS2 intron 1, Venus: Venus fluorescent protein (a YFP variant), FLAG: triple-FLAG tag, |: stop codon, *: bicistronic intergenic sequence (tagcat), AphVIII ${ }^{\mathrm{R}}$ : Paromomycin resistance gene, $\mathrm{R}^{\mathrm{T}}$ : RBCS 2 terminator. Candidate peptides are Rubisco activase (RBCA, A-E) and Rubisco small subunit (RBCS, B-J) chloroplast targeting peptides (cTP) extended by $0,3,5,10$ or 23 residues beyond the cleavage site into the mature protein, as well as carbonic anhydrase 4 (CAH4, K) and $\gamma$-carbonic anhydrase 2 (CAG2, L) mitochondrial targeting peptides (mTP). Lengths in amino acid residues are indicated above each peptide. Introns within peptides are symbolized by dotted lines. Typical epifluorescence images of resulting transformants in strain $\mathrm{T}_{22} 22^{+}$show fluorescence from the Venus channel in yellow and chlorophyll autofluorescence in magenta. Confocal microscopy images include an additional MitoTracker channel in cyan for mTPs. All scale bars are $5 \mu \mathrm{m}$ in length. 


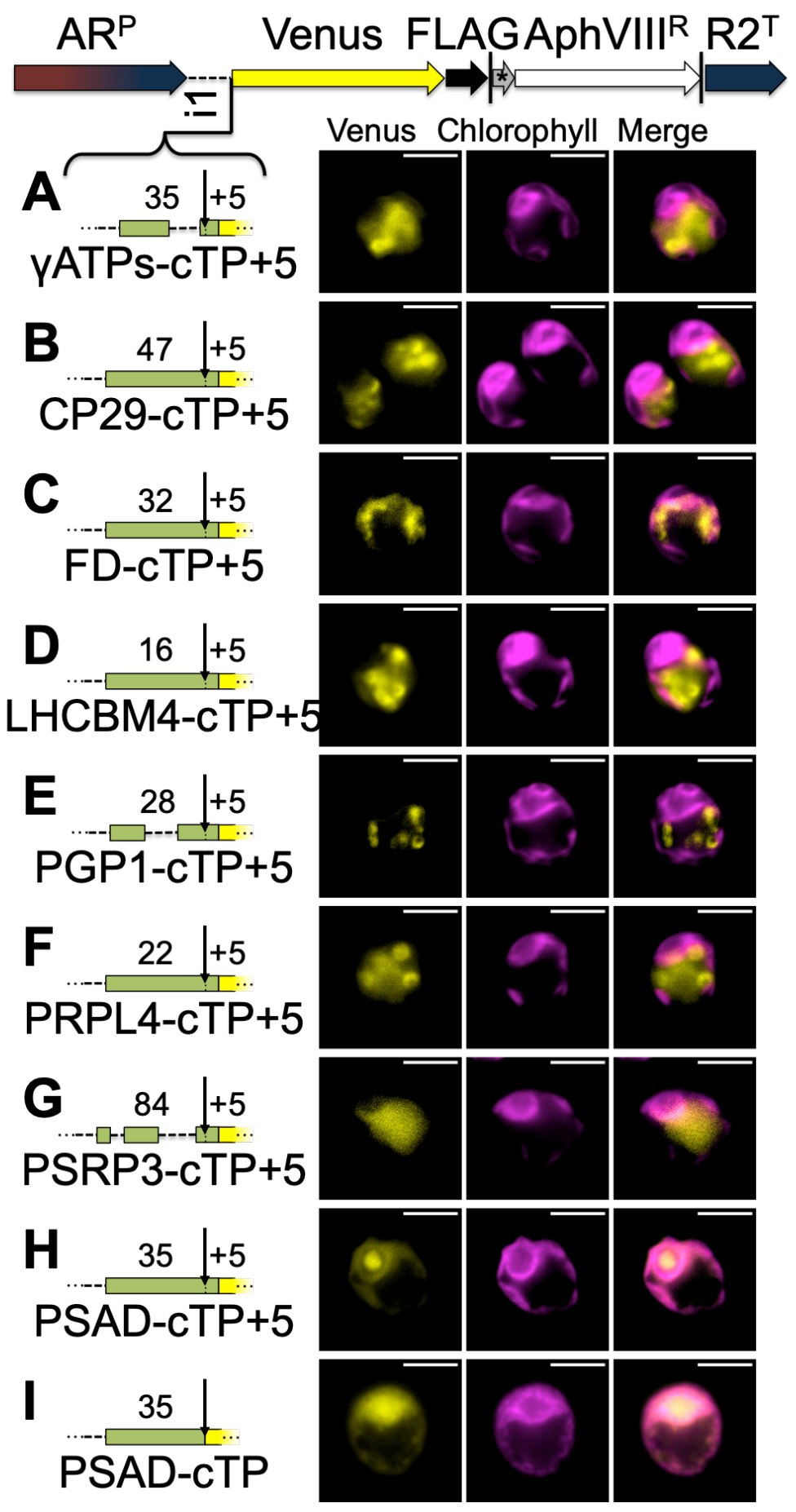

Fig. 2 - Without a stretch of post-cleavage site residues, Chlamydomonas chloroplast targeting peptides fail to import. Candidate chloroplast targeting peptides, each including 5 post-cleavage site residues, from (A) chloroplast ATP-synthase gamma chain ( $\gamma$ ATPs), (B) chlorophyll a/b binding protein of photosystem II (CP29), (C) ferredoxin (FD), (D) chlorophyll a/b binding protein of

316 light harvesting complex II (LHCBM4), (E) phosphoglycolate phosphatase (PGP1), (F) plastid ribosomal protein L4 (PRPL4), (G) plastid-specific ribosomal protein 3 (PSRP3) and (H) Photosystem 1 subunit D (PSAD), were inserted in-frame upstream of Venus. (I) A second PSAD construct lacking post-cleavage site residues was probed. Lengths in amino acid residues are

320 indicated above each peptide. Representation as in Fig. 1. 
Intrinsically
disordered protein segment

$\mathrm{AR}^{\mathrm{P}}$ Venus FLAGAphVIIIR R2'

\title{
B
}

\author{
Unstructured \\ C-terminal
} protein segment
C

$$
\begin{aligned}
& \text { Artificial } \\
& \text { G-rich linke } \\
& \text { sequence }
\end{aligned}
$$ G-rich linker
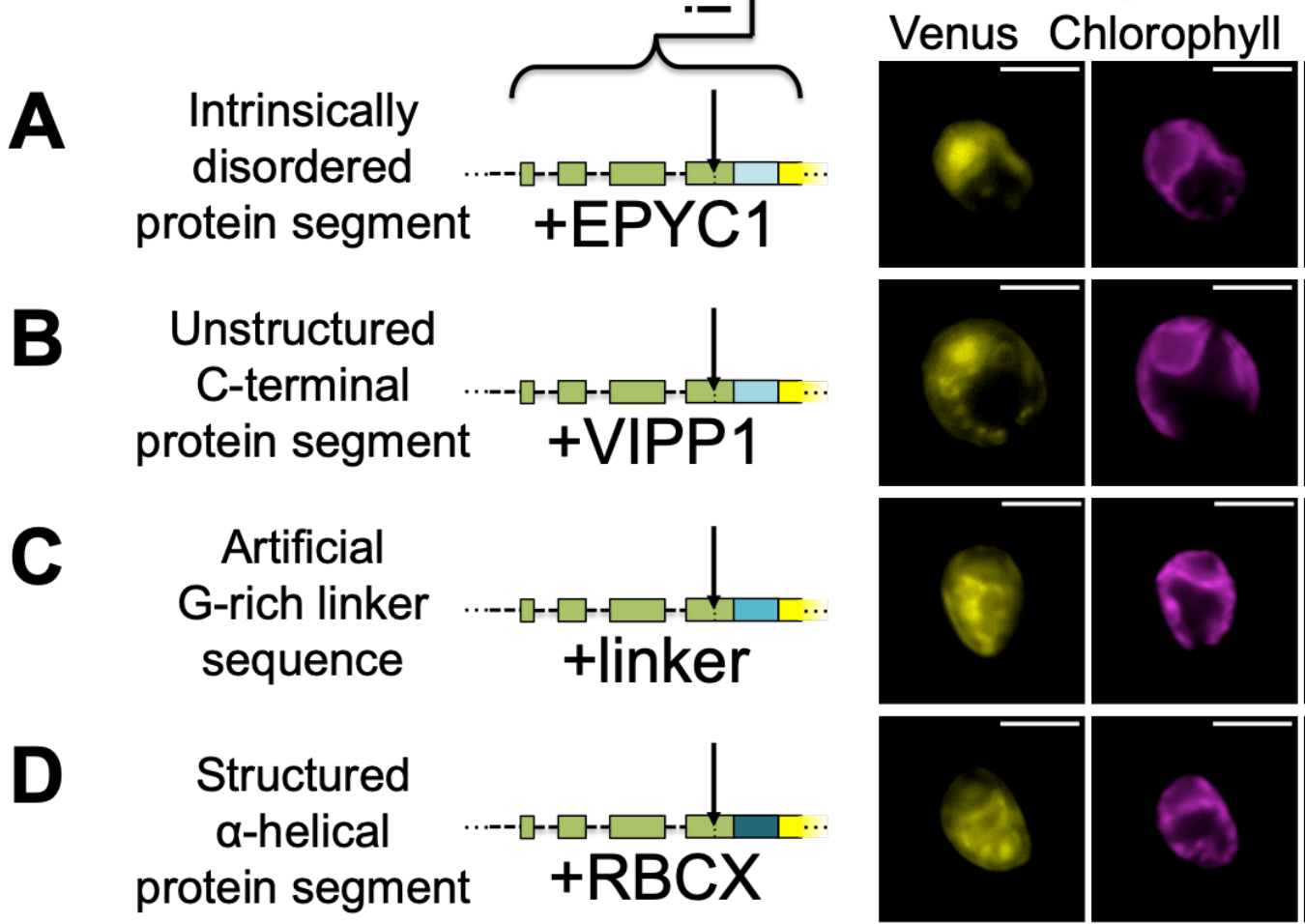

Merge
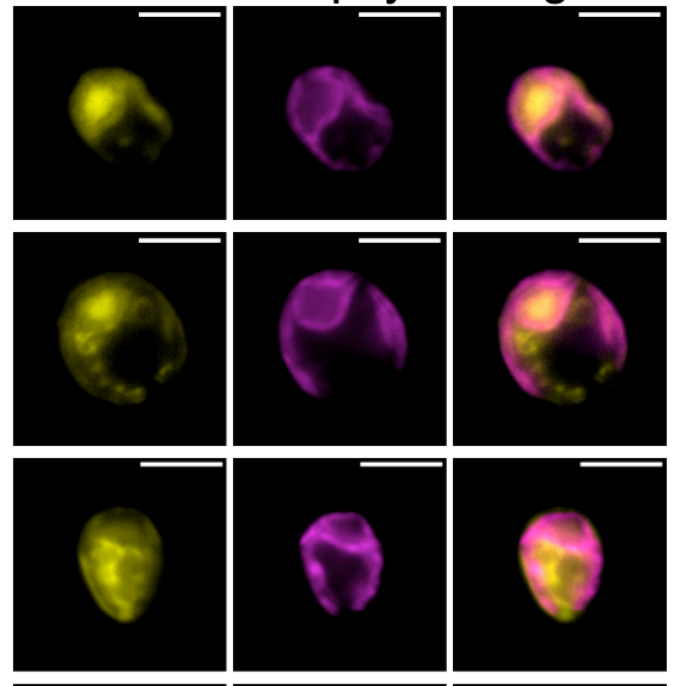

Fig. 3 - Unstructured downstream sequence aids cTP targeting. The following candidate sequences were inserted downstream of RBCA-cTP+5 and upstream of Venus: (A) residues 195-213 of intrinsically disordered protein EPYC1, which are part of the $3^{\text {rd }}$ out of 4 repeats; (B) residues 260278 of VIPP1, which form part of an unstructured C-terminus; (C) an artificial linker sequence mainly consisting of quintuple-G interspersed with S; (D) residues 135-154 of RBCXa, which form part of the most C-terminal $\alpha$-helix. Refer to Table 1 for amino acid sequences. Representation as in

328 Fig. 1.

\section{Tables}

330 Table 1 - Amino acid sequences. Cleavage sites are indicated with a full stop between residues.

Element

RBCS-cTP (+23)

RBCA-cTP (+23)
MAAVIAKSSVSAAVARPARSSVRPMAALKPAVKAAPV

AAPAQANQ.MMVWTPVNNKMFETFSYLPPLSD

Amino acid sequence

MQVTMKSSAVSGQRVGGARVATRSVRRAQLQVV.ASS

RKQMGRWRSIDAGVDASDDQ

CAH4-mTP

MSSRNVATALRMFATLGRSQAGEASAMMGTGSALLA QRAAALGGASAVNKGCSCRCGRVACMGACMPMRH. 


\section{CAG2-mTP MLKRVGQSLVPFARAGLTQTAESFRG.}

$\boldsymbol{\gamma}$ ATPs-cTP (+5) MAAMLASKQGAFMGRSSFAPAPKGVASRGSLQVVA.G LKEV

CP29-cTP (+5) MVFKFPTPPGTQKKAGTTATKPAPKATTKKVATSTGTR SGGVGYRKY.QGDAL

FD-cTP (+5) MAMAMRSTFAARVGAKPAVRGARPASRMSCMA.YKV TL

LHCBM4-cTP (+5) MAFALATSRKALQVTC.KATGK

PGP1-cTP (+5) MVAAQASARPIATNEQKLELLKKVECFI.FDCDG

PRPL4-cTP (+5) MQTMRVAFRPAATSRSTVVTRA.SAVAA

PSRP3-cTP (+5) MMLVRQVPCLAQRASARSAVRSRPAPFTPARRVFARA QADAALLEDDEVAYEVTNEDFELDENDPDLLLGLEAV ELMGSDVDAM.TAAAV

PSAD-cTP (+5) MAVMMRTQAPAATRASSRVAVAARPAARRAVVVRA. EAEAA

+ EPYC1

SSSPAPASSAPAPARSSSA

+VIPP1 SAVGSLPEPRAVDALDLEL

+linker $\quad$ SGGGGSGGGGSMGGGGSN

+ RBCX KTMEMASRETREANTRLM 